Ophthalmologe 2019 $\cdot 116: 815-816$

https://doi.org/10.1007/s00347-019-0914-2

(c) Springer Medizin Verlag GmbH, ein Teil von Springer Nature 2019

\section{Claus Cursiefen}

Zentrum für Augenheilkunde, Uniklinik Köln, Köln, Deutschland

\title{
Augenheilkunde: unser Fach mit Zukunft
}

Das Sehen ist die wichtigste Sinnesfunktion des Menschen. Etwa ein Drittel des menschlichen Gehirns ist mit der Verarbeitung von Sinnesinformationen beschäftigt. Wenn man Menschen nach Angst vor Erkrankungen befragt, ist die Angst vor Erblindung immer ganz weit oben, weit vor der Angst z. B. vor kardiovaskulären Erkrankungen [1]. Für viele von uns ist die Augenheilkunde unter anderem auch deshalb das „schönste Fach innerhalb der Medizin“, weil wir vielen unserer Patienten helfen können, lange und gut $\mathrm{zu}$ sehen.

\section{) Die Augenheilkunde ist eine Teildisziplin der Medizin mit stetig wachsendem Versorgungsauftrag}

Im Vergleich zu anderen Fachdisziplinen gilt die Augenheilkunde oft dennoch als „kleines“Fach in der Medizin. Dies völlig zu Unrecht, da wir mit Erkrankungen wie dem grauen Star, dem trockenen Auge, dem Glaukom, der altersabhängigen Makuladegeneration (AMD) oder auch Hornhautdystrophien viele „ophthalmologische Volkskrankheiten“ behandeln. Daneben ist die Augenheilkunde auch eine enorm wichtige, erfolgreiche und wertgeschätzte Teildisziplin der Medizin mit stetig wachsendem Versorgungsauftrag. Das dürfen und sollen wir - v. a. im Interesse unserer aktuellen und zukünftigen Patienten - auch immer wieder mal nach außen tragen, um die Relevanz und Attraktivität unseres „kleinen“ Faches gerade auch im Wettstreit um engagierten Nachwuchs, Forschungsfördermittel oder andere Ressourcen im Gesundheitswesen zu untermauern.
Ziel dieses Themenheftes soll gemäß dem Motto des 117. Kongresses der DOG (Deutsche Ophthalmologische Gesellschaft) in Berlin deshalb eine aktuelle Standortbestimmung des Faches Augenheilkunde als „unser Fach mit Zukunft“ sein. Dabei blicken wir zum einen zurück, betrachten die Gegenwart und schauen in die Zukunft.

Den Blick zurück beginnt Herr Prof. Rohrbach von der Universitätsaugenklinik Tübingen mit seinem Beitrag „Zukunft durch Geschichte“. Die Gestaltung der Zukunft setzt naturgemäß Kenntnis der Vergangenheit voraus. Anhand verschiedener Beispiele stellt Herr Prof. Rohrbach hier Entwicklungslinien in der Augenheilkunde in der Vergangenheit mit Auswirkung für die Gegenwart und die Zukunft sehr anschaulich und gut bebildert zusammen.

Es schließt sich an ein Beitrag von Prof. Schuster von der Universitätsaugenklinik in Mainz zum Thema „Augenheilkunde 2019 - wo stehen wir? Eine Betrachtung der Versorgungssituation in Deutschland“. Schuster et al. beleuchten hier die aktuelle Versorgungssituation von Patienten mit Augenerkrankungen in Deutschland, z. T. basiert auf nationalen Kohortenstudien, den stetig steigenden Versorgungsbedarf in der Augenheilkunde und die Implikationen, die sich daraus für die Zukunft ergeben.

Abschließend folgt ein Beitrag, der in Zusammenarbeit mit dem European Vision Institut (EVI) in Brüssel als Consensus Road Map erstellt wurde. Der Beitrag mit dem Titel „Zukünftige Herausforderungen in der ophthalmologischen Forschung" basiert auf einem englischen Beitrag zu dem Thema „Unmet needs in ophthalmic research: an EVI consensus road map 2019-2025“. Hier 
wird in verschiedenen Teildisziplinen der Augenheilkunde aufgezeigt, welche patientenrelevanten Fragestellungen in der Forschung in den nächsten Jahren realistischerweise adressiert werden können und sollen. Dieser Beitrag dient als Argumentationshilfe gegenüber Politik, (pharmazeutischer) Industrie/Wirtschaft und Patientenverbänden.

Zusammenfassend hoffe ich, dass die Beiträge dieses Themenheftes Ihnen und auch fachfremden Lesern zeigen, welchen Stellenwert die Augenheilkunde im medizinischen Fächerkanon und natürlich vor allen Dingen für unsere Patienten hat, wie historische Entwicklungen uns heute und in Zukunft beeinflussen, welchen aktuellen Versorgungsbedingungen wir exponiert sind und wie der Weg unseres Faches in Zukunft weitergehen kann, um die Versorgung unserer Patienten auch u. a. durch Forschungsanstrengungen noch weiter zu verbessern. Wir hoffen, damit einen Beitrag zum Thema „Augenheilkunde - unser Fach mit Zukunft“ geleistet zu haben.

Ich wünsche Ihnen einen interessanten und erfreulichen Kongress in Berlin und eine anregende Lektüre! Mit freundlichen Grüßen aus Köln

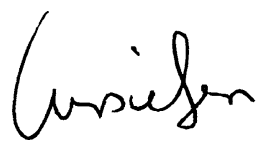

Univ.-Prof. Dr. med. C. Cursiefen Direktor des Zentrums für Augenheilkunde

\section{Korrespondenzadresse

\begin{tabular}{l} 
Univ.-Prof. Dr. med. \\
Claus Cursiefen, FEBO, \\
FARVO \\
Zentrum für Augenheilkunde, \\
$\begin{array}{l}\text { Uniklinik Köln } \\
\text { Kerpener Str. 62, 50937 Köln, } \\
\text { Deutschland } \\
\text { claus.cursiefen@uk-koeln.de }\end{array}$ \\
\hline
\end{tabular}

Interessenkonflikt. C. Cursiefen gibt an, dass kein Interessenkonflikt besteht.

\section{Literatur}

1. Scott AW, Bressler N, Ffolkes S, Wittenborn JS, JorkaskyJ (2016) Public attitudes about eye and vision health. JAMA Ophthalmol 1;134(10):1111-1118

In eigener Sache

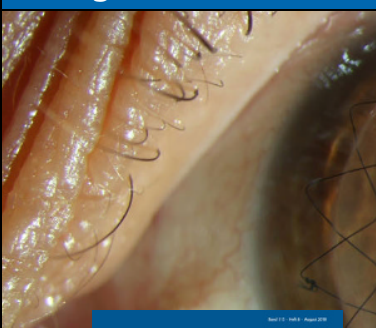

Der Ophthalmologe
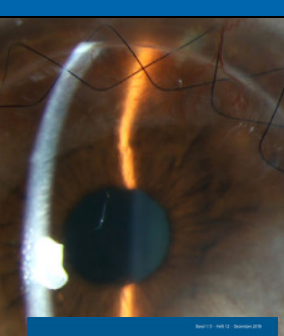

Der Ophthalmologe

Der Ophthalmologe
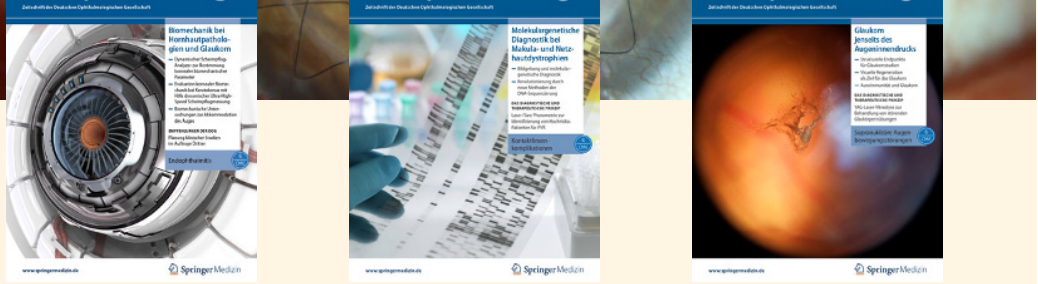

\section{Leitthemenübersicht von Der Ophthalmologe}

Der Ophthalmologe bietet Ihnen jeden Monat umfassende und aktuelle Beiträge zu interessanten Themenschwerpunkten aus allen Bereichen der Augenheilkunde.

Rückblick - 2018

01/18 Originalien

02/18 Aktuelles zum trockenen Auge

03/18 Nanomedizin

04/18 Lidchirurgie

05/18 Minimalinvasive Glaukomchirurgie

06/18 Frühgeborenenretinopathie

07/18 Elektronische Hilfsmittel für

Sehbehinderte

08/18 Biomechanik bei

Hornhautpathologien und Glaukom

09/18 Deep Learning

10/18 Versorgungsforschung

11/18 Kantenfiltergläser

12/18 Molekulargenetische Diagnostik bei

Makula- und Netzhautdystrophien

Alle Inhalte der Zeitschrift finden Sie unter

www.springermedizin.de/

Der-Ophthalmologe
Rückblick \& Vorschau - 2019

01/19 Glaukom jenseits des

Augeninnendrucks

02/19 Neurotrophe Keratopathie

03/19 Therapie der Fuchs-Endothel-

dystrophie mittels DMEK

04/19 Iristumoren

05/19 Komplexe Glaukome mit hohem

Behandlungsrisiko

06/19 Myopie - Epidemiologie,

Pathomechanismen und Prävention

07/19 Originalien

08/19 OCT-Angiographie und systemische Erkrankungen

09/19 Augenheilkunde: unser Fach mit

Zukunft

10/19 Schwere Flüssigkeiten in der

Netzhautchirurgie

11/19 Makulaformen und andere

Makulopathien

12/19 Augenverletzungen durch

Feuerwerks- und Knallkörper

(Änderungen vorbehalten)

SpringerMedizin.de bietet Ihnen Zugang zu allen elektronisch verfügbaren Ausgaben Ihrer Zeitschrift - unabhängig davon, seit wann Sie die Zeitschrift abonniert haben. 